\title{
A PROCEDURE FOR CHARACTERIZING THE RANGE OF INPUT UNCERTAINTY PARAMETERS BY THE USE OF THE FFTBM
}

\author{
A. Petruzzi, A. Kovtonyuk, M. Raucci, D. De Luca, F. Veronese and F. D’Auria \\ Nuclear Research Group of San Piero a Grado, University of Pisa, Pisa, Italy
}

\begin{abstract}
In the last years various methodologies were proposed to evaluate the uncertainty of Best Estimate (BE) code predictions. The most used method at the industrial level is based upon the selection of input uncertain parameters, on assigning related ranges of variations and Probability Distribution Functions (PDFs) and on performing a suitable number of code runs to get the combined effect of the variations on the results.
\end{abstract}

A procedure to characterize the variation ranges of the input uncertain parameters is proposed in the paper in place of the usual approach based (mostly) on engineering judgment. The procedure is based on the use of the Fast Fourier Transform Based Method (FFTBM), already part of the Uncertainty Method based on the Accuracy Extrapolation (UMAE) method and extensively used in several international frameworks.

The FFTBM has been originally developed to answer questions like "How long improvements should be added to the system thermal-hydraulic code model? How much simplifications can be introduced and how to conduct an objective comparison?”. The method, easy to understand, convenient to use and user independent, clearly indicates when simulation needs to be improved.

The procedure developed for characterizing the range of input uncertainty parameters involves the following main aspects:

a) One single input parameter shall not be 'responsible' for the entire error |exp-calc|, unless exceptional situations to be evaluated case by case;

b) Initial guess for Max and Min for variation ranges to be based on the usual (adopted) expertise;

c) More than one experiment can be used per each NPP and each scenario. Highly influential parameters are expected to be the same. The bounding ranges should be considered for the NPP uncertainty analysis;

d) A data base of suitable uncertainty input parameters can be created per each NPP and each transient scenario. 


\section{Introduction}

Several approaches have been proposed to quantify the accuracy of a given code calculation ${ }^{1,2,3,4}$. Even though these methods were able to give some information about the accuracy, they were not considered satisfactory because they involved some empiricism and were lacking of a precise mathematical meaning. Besides, engineering subjective judgment at various levels is deeply inside in proposed methods.

Generally, the starting point of each method is an error function, by means of which the accuracy is evaluated. Some requirements were fixed which an objective error function should satisfy:

- at any time of the transient this function should remember the previous history;

- engineering judgment should be avoided or reduced;

- the mathematical formulation should be simple;

- the function should be non-dimensional;

- it should be independent upon the transient duration;

- compensating errors should be taken into account (or pointed out);

- its values should be normalized.

The Fast Fourier Transform Based Method (FFTBM) has been developed taking into account the above requirements and to answer questions like "How long improvements should be added to the system thermal-hydraulic code model? How much simplifications can be introduced and how to conduct an objective comparison?”. The method, easy to understand, convenient to use and user independent, clearly indicates when simulation needs to be improved.

The objective of the paper is to propose a procedure to characterize the ranges of variation of the input uncertain parameters in place of the usual approach based (mostly) on engineering judgment for the uncertainty methods based on the propagation of input error. The proposed procedure is based on an alternative way to use the FFTBM respect to its common application inside the UMAE approach.

In order to give a full picture about the framework where the FFTBM is used, in section 2 a short description about the approaches to perform uncertainty analysis is given. More details about the methods that need the FFTBM and the existing interconnections between these tools is provided in section 3, whereas section 4 contains the description of the FFTBM. Section 5 presents the use of the FFTBM as a tool for characterizing the ranges of variation of the input uncertain parameters.

\section{Approaches for performing Uncertainty Analysis}

The features of two independent approaches for estimating uncertainties are reviewed below. The propagation of code input errors (Fig. 1): this can be evaluated as being the most adopted procedure nowadays, endorsed by industry and regulators. It adopts the statistical combination of values from selected input uncertainty parameters (even though, in principle an unlimited number of input parameters can be used) to calculate the propagation of the errors throughout the code. To this approach belongs the so-called "GRS method" 5 and the majority of methods adopted by the nuclear industry. Although the entire set of the actual number of input parameters for a typical NPP (Nuclear Power Plant) input deck, ranging up to about $10^{5}$ input parameters, could theoretically be considered as uncertainty sources by these methods, only a 'manageable' number (of the order of several tens) is actually taken into account in 
practice. Ranges of variations, together with suitable PDF (Probability Density Function) are then assigned for each of the uncertain input parameter actually considered in the analysis. The number of computations needed for obtaining the desired confidence in the results can be determined theoretically by the Wilks formula ${ }^{6}$. Subsequently, the identified computations (ca. 100) are performed using the code under investigation to propagate the uncertainties inside the code, from inputs to outputs (results). The logical steps of the approach are depicted in Fig. 1.

The main drawbacks of such methods are connected with: (i) the need of engineering judgment for limiting (in any case) the number of the input uncertain parameters; (ii) the need of engineering judgment for fixing the range of variation and the PDF for each input uncertain parameter; (iii) the use of the codenodalization for propagating the uncertainties: if the code-nodalization is wrong, not only the reference results are wrong but also the results of the uncertainty calculations and (iv) the process of selecting the (about) 100 code runs is not convergent.

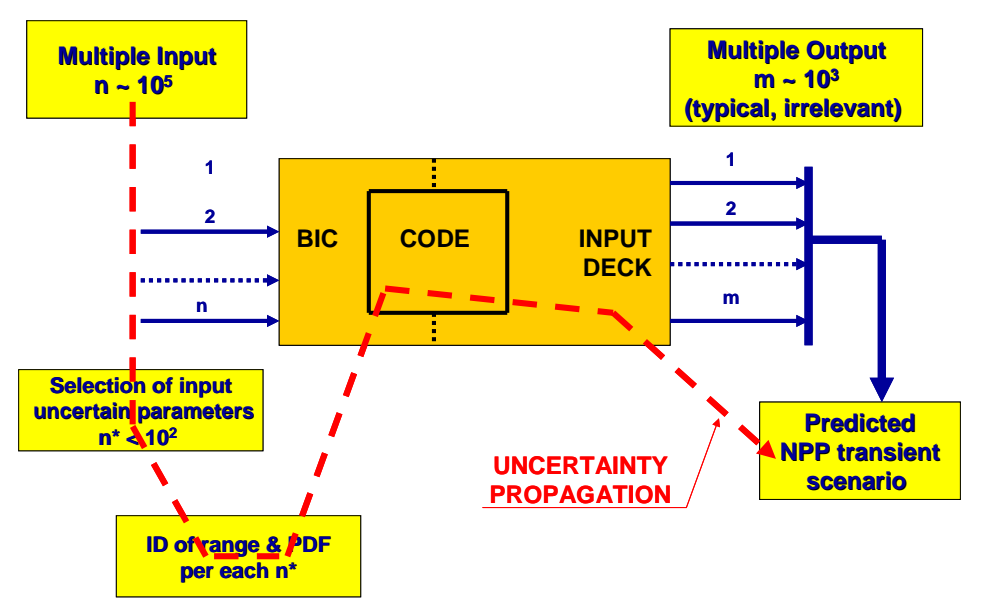

Figure 1. Uncertainty methods based upon propagation of input uncertainties (GRS method).

The second approach (Fig. 2), reviewed as the propagation of code output errors, is representatively illustrated by the UMAE-CIAU (Uncertainty Method based upon Accuracy Extrapolation ${ }^{7}$ 'embedded' into the Code with capability of Internal Assessment of Uncertainty ${ }^{8,9}$ ). Note that this class of methods includes only a few applications from industry. The use of this method depends on the availability of 'relevant' experimental data, where here the word 'relevant' is connected with the specific NPP transient scenario under investigation for uncertainty evaluation. Assuming such availability of relevant data, which are typically Integral Test Facility (ITF) data, and assuming the code correctly simulates the experiments, it follows that the differences between code computations and the selected experimental data are due to errors. If these errors comply with a number of acceptability conditions ${ }^{7}$, then the resulting (error) database is processed and the 'extrapolation' of the error takes place. Relevant conditions for the extrapolation are:

- Building up the NPP nodalization with the same criteria as was adopted for the ITF nodalizations;

- Performing a similarity analysis and demonstrating that NPP calculated data are "consistent" with the data measured in a qualified ITF experiment. 


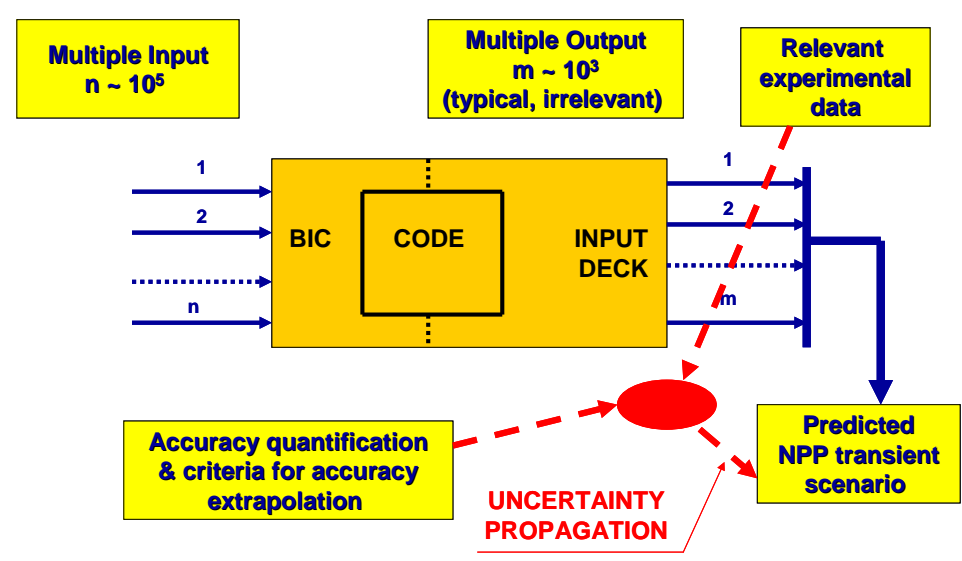

Figure 2. Uncertainty methods based upon propagation of output uncertainties (CIAU method).

The main drawbacks of this method are as follows: (i) the method is not applicable in the absence of relevant experimental information; (ii) a considerable amount of resources is needed to establish a suitable error database, but this is a one-time effort, independent of subsequent applications of this method; (iii) the process of combining errors originating from different sources (e. g, stemming from different ITF or SETF (Separate Effect Test Facility), different but consistent nodalizations, different types of transient scenarios) is not based upon fundamental principles and requires detailed validation.

The FFTBM procedure here proposed to characterize the ranges of variation of the input uncertain parameters is mostly connected with the second approach (i.e. the propagation of output uncertainties).

\section{UMAE-CIAU Method}

\subsection{The UMAE Methodology: the Engine of the CIAU Method}

The UMAE", whose flow diagram is given in Fig. 3, is the prototype method for the description of "the propagation of code output errors" approach. The method focuses not on the evaluation of individual parameter uncertainties but on the propagation of errors from a suitable database calculating the final uncertainty by extrapolating the accuracy from relevant integral experiments to full scale NPP. Considering ITF of reference water cooled reactor, and qualified computer codes based on advanced models, the method relies on code capability, qualified by application to facilities of increasing scale. Direct data extrapolation from small scale experiments to reactor scale is difficult due to the imperfect scaling criteria adopted in the design of each scaled down facility. So, only the accuracy (i.e. the difference between measured and calculated quantities) is extrapolated.

Experimental and calculated data in differently scaled facilities are used to demonstrate that physical phenomena and code predictive capabilities of important phenomena do not change when increasing the dimensions of the facilities (see right loop FG in Fig. 6). Other basic assumptions are that phenomena and transient scenarios in larger scale facilities are close enough to plant conditions. The influence of user and nodalizations upon the output uncertainty is minimized in the methodology. However, user and nodalization inadequacies affect the comparison between measured and calculated trends; the error due to this is considered in the extrapolation process and gives a contribution to the overall uncertainty. 


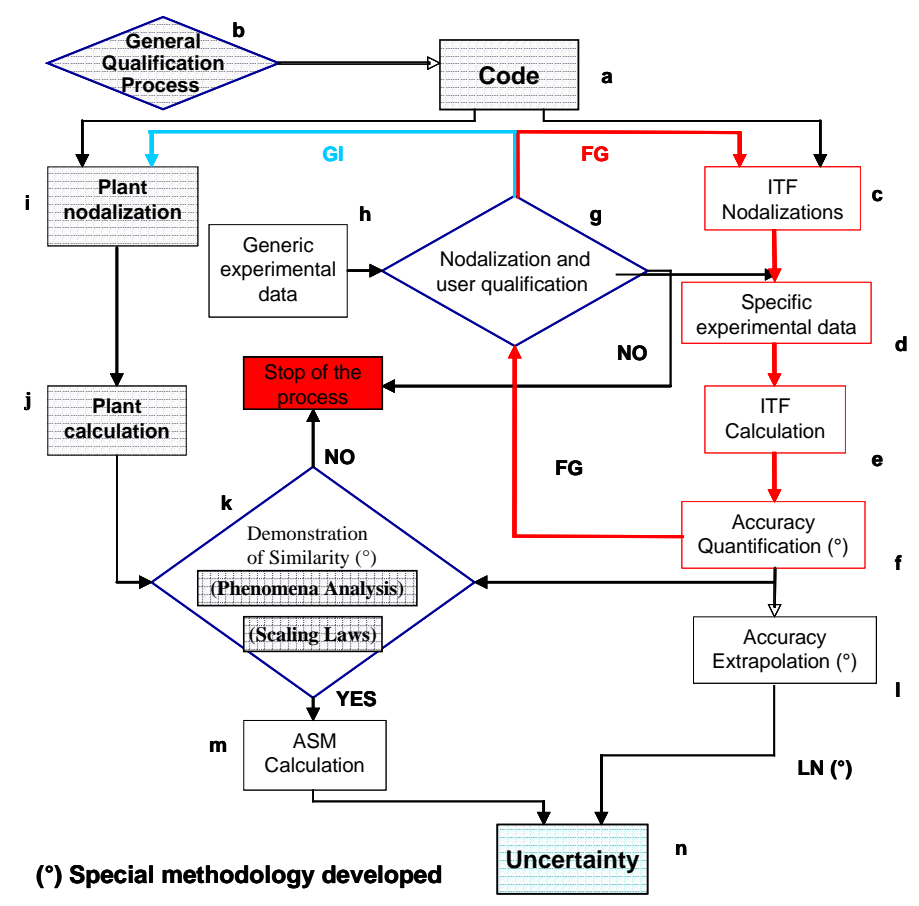

Figure 3. UMAE flow diagram (also adopted within the process of application of CIAU).

The method utilizes a database from similar tests and counterpart tests performed in ITF, that are representative of plant conditions. The quantification of code accuracy (step 'f' in Fig. 3) is carried out by using a procedure based on the Fast Fourier Transform Based Method (FFTBM ${ }^{10}$ ) characterizing the discrepancies between code calculations and experimental data in the frequency domain, and defining figures of merit for the accuracy of each calculation. Different requirements have to be fulfilled in order to extrapolate the accuracy. Calculations of both ITF experiments and NPP transients are used to attain uncertainty from accuracy. Nodalizations are set up and qualified against experimental data by an iterative procedure, requiring that a reasonable level of accuracy is satisfied. Similar criteria are adopted in developing plant nodalization and in performing plant transient calculations (see left loop FG in Fig. 6). The demonstration of the similarity of the phenomena exhibited in test facilities and in plant calculations, accounting for scaling laws considerations (step ' $k$ ' in Fig. 6), leads to the Analytical Simulation Model (ASM), i.e. a qualified nodalization of the NPP.

\subsection{The CIAU Method}

The basic idea of the CIAU ${ }^{8,9}$ can be summarized in two parts:

- Consideration of plant status: each status is characterized by the value of six "driving” quantities (their combination is the "hypercube") and by the time instant when those values are reached during the transient;

- Association of uncertainty (quantity and time) to each plant status. 
A key feature of CIAU is the full reference to the experimental data (see loop FG in Fig. 3). Accuracy from the comparison between experimental and calculated data is extrapolated to obtain uncertainty. A solution to the issues constituted by the "scaling" and "the qualification" of the computational tools is embedded into the method ${ }^{11,12}$ through the UMAE methodology that constitutes the engine for the development of CIAU and for the creation of the error database.

Assigned a point in the time domain, the accuracy in predicting the time of occurrence of any point is distinguished from the accuracy that characterizes the quantity value at that point. Thus, the time-domain and the phase-space are distinguished: the time-domain is needed to characterize the system evolution (or the NPP accident scenario) and the phase-space domain is used to identify the hypercubes.

Quantity and time accuracies are associated to errors-in-code-models and uncertainties-in-boundary-andinitial-conditions including the time sequence of events and the geometric model of the problem. Thus,

a) The 'transient-time-dependent' calculation by a code resembles a succession of steady-state values at each time step and is supported by the consideration that the code is based on a number and a variety of empirical correlations qualified at steady-state with assigned geometric discretization. Therefore, quantity accuracy can be associated primarily with errors-in-code-models.

b) Error associated with the opening of a valve (e.g. time when the equivalent full flow area for the flow passage is attained) or inadequate nodalization induce time errors that cannot be associated to code model deficiencies. Therefore, time accuracy can be associated primarily with uncertainties-inboundary-and-initial-conditions.

Once the Time Accuracy (Uncertainty) Vector, TAV (TUV), and the Quantity Accuracy (Uncertainty) Matrix, QAM (QUM) are derived, the overall accuracy (and uncertainty) is obtained by the geometric combination of the two accuracies (and uncertainties) values, i.e. time and quantity, in the twodimensional space-time plane.

An idea of the architecture of the CIAU methodology can be derived from Fig. 4. Two processes can be distinguished: the "Error Filling Process" (similar to path FG in Figure 3) by which the NPP statuses are filled with the values of the error database, and the "Error Extraction Process" by which the uncertainty values (derived from the extrapolation process of accuracy) are picked up from the NPP statuses.

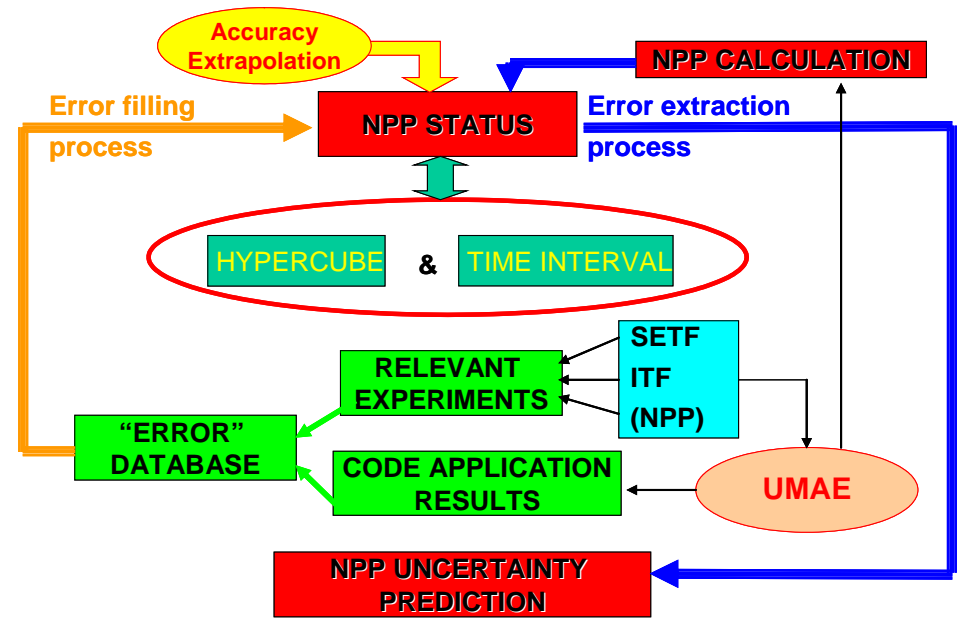

Figure 4. CIAU Method: “Error Filling Process” and “Error Extraction Process”. 


\section{The FFTBM Tool}

The simplest formulation about the accuracy of a given code calculation, with reference to the experimental measured trend, is obtained by the difference function:

$$
\Delta F(t)=F_{\text {calc }}(t)-F_{\text {exp }}(t)
$$

The information contained in this time dependent function, continuously varying, should be condensed to give a limited number of values which could be taken as indexes for quantifying accuracy. This is allowed because the complete set of instantaneous values of $\mathrm{DF}(\mathrm{t})$ is not necessary to draw an overall judgment about accuracy.

Integral approaches satisfy this requirement, since they produce a single value on the basis of the instantaneous trend of a given function of time. On the other hand, searching for functions expressing all the information through a single value, some interesting details could be lost. Therefore, it would be preferable to define methodologies leading to more than one value in order to characterize the code calculation accuracy.

Information that comes from the time trend of a certain parameter, be it a physical or a derivate one, may be not sufficient for a deep comprehension of a concerned phenomenon; in such a case, it may be useful to study the same phenomenon from other points of view, free of its time dependence. In this context, the complete behaviour of a system in periodic regime conditions (periodic conditions due to instability phenomena are explicitly excluded), can be shown by the harmonic response function that describes it in the frequency domain.

Furthermore, the harmonic analysis of a phenomenon can point out the presence of perturbations otherwise hidden in the time domain.

\subsection{The FFT algorithm}

It is well known that the Fourier transform is essentially a powerful problem solving technique. Its importance is based on the fundamental property that one can analyze any relationship from a completely different viewpoint, with no lack of information with respect to the original one. The Fourier transform can translate a given time function $\mathrm{g}(\mathrm{t})$, in a corresponding complex function defined, in the frequency domain, by the relationship:

$$
\tilde{g}(f)=\int_{-\infty}^{+\infty} g(t) \cdot e^{-j \cdot 2 \pi \cdot f \cdot t} d t
$$

Afterwards, it is assumed that the experimental and calculated trends, to which the Fourier transform is applied, verify the analytical conditions required by its application theory; i.e., it is assumed that they are continuous (or generally continuous) ${ }^{(1)}$ in the considered time intervals with their first derivatives, and

(1) : i.e. discontinuous only in a finite number of points. The existence of the Fourier Transform is guaranteed if $g(t)$ is summable according to Lebesgue on the real axis. 
absolutely integrable in the interval ${ }^{13}(-\infty,+\infty)^{(2)}$. This last requirement can be easily satisfied in our case, since the addressed functions assume values different from zero only in the interval $(0, T)$. Therefore:

$$
\tilde{g}(f)=\int_{0}^{T} g(t) \cdot e^{-j \cdot 2 \pi \cdot f \cdot t} d t
$$

The Fourier integral is not suitable for machine computation, because an infinity of samples of $g(t)$ is required. Thus, it is necessary to truncate the sampled function $g(t)$ so that only a finite number of points are considered, or in other words, the discrete Fourier transform is evaluated. Truncation introduces a modification of the original Fourier transform (the Fourier transform of the truncated $\mathrm{g}(\mathrm{t})$ has a rippling); this effect can be reduced choosing the length of the truncation function as long as possible.

When using functions sampled in digital form, the Fast Fourier Transform (FFT) can be used. The FFT is an algorithm that can compute more rapidly the discrete Fourier transform. To apply the FFT algorithm, functions must be identified in digital form by a number of values which is a power of 2 . Thus, if the number of points defining the function in the time domain is:

$$
N=2^{m+1}
$$

the algorithm gives the transformed function defined in the frequency domain by $2^{\mathrm{m}}+1$ values corresponding to the frequencies $f_{n}$, in which $T$ is the time duration of the sampled signal:

$$
\mathrm{f}_{\mathrm{n}}=\frac{\mathrm{n}}{\mathrm{T}} \quad\left(\mathrm{n}=0,1, \ldots, 2^{\mathrm{m}}\right)
$$

Taking into account the fact that the adopted subroutine packages evaluate the FFT normalized to the time duration T, from Eqs. (3) and (5) it can be seen that $|\tilde{g}(0)|$ represents the mean value of the function $g(t)$ in the interval $(0, \mathrm{~T})$, while $\left|\tilde{g}\left(f_{i}\right)\right|$ represents the amplitude of the $i$-th term of the Fourier polynomial expansion for the function $\mathrm{g}(\mathrm{t})$. Generally, the Fourier transform is a complex quantity described by the following relationship:

$$
\tilde{g}(f)=\operatorname{Re}(f)+j \cdot \operatorname{Im}(f)=|\tilde{g}(f)| \cdot e^{j \theta(f)}
$$

where:

- $\operatorname{Re}(\mathrm{f})$ is the real component of the Fourier transform

- $\operatorname{Im}(\mathrm{f})$ is the imaginary component of the Fourier transform

- $|\tilde{g}(f)|$ is the amplitude or Fourier spectrum of $\mathrm{g}(\mathrm{t})$

- $\theta(f)$ is the phase angle or phase spectrum of Fourier transform. It is well known that:

(2) : i.e. $\int_{-\infty}^{+\infty}|g(t)| d t<\infty$ 


$$
\begin{gathered}
|\tilde{g}(f)|=\sqrt{(\operatorname{Re}(f))^{2}+(\operatorname{Im}(f))^{2}} \\
\theta(\mathrm{f})=\operatorname{tg}^{-1} \frac{\operatorname{Im}(\mathrm{f})}{\operatorname{Re}(\mathrm{f})}
\end{gathered}
$$

\subsection{The FFTBM algorithm}

The method developed to quantify the accuracy of code calculations is based on the amplitude of the FFT of the experimental signal and of the difference between this one and the calculated trend. In particular, with reference to the error function $\Delta \mathrm{F}(\mathrm{t})$, defined by the Eq. (1), the method defines two values characterizing each calculation:

\section{The Dimensionless Average Amplitude, AA:}

$$
\mathrm{AA}=\frac{\sum_{\mathrm{n}=0}^{2^{\mathrm{m}}}\left|\widetilde{\Delta} \mathrm{F}\left(\mathrm{f}_{\mathrm{n}}\right)\right|}{\sum_{\mathrm{n}=0}^{2^{\mathrm{m}}}\left|\widetilde{\mathrm{F}}_{\exp }\left(\mathrm{f}_{\mathrm{n}}\right)\right|}
$$

\section{The Weighted Frequency, WF:}

$$
\mathrm{WF}=\frac{\sum_{\mathrm{n}=0}^{2^{\mathrm{m}}}\left|\tilde{\Delta} \mathrm{F}\left(\mathrm{f}_{\mathrm{n}}\right)\right| \cdot \mathrm{f}_{\mathrm{n}}}{\sum_{\mathrm{n}=0}^{2^{\mathrm{m}}}\left|\tilde{\Delta} \mathrm{F}\left(\mathrm{f}_{\mathrm{n}}\right)\right|}
$$

The AA factor can be considered a sort of "average fractional error" of the addressed calculation, whereas the weighted frequency WF gives an idea of the frequencies related with the inaccuracy ${ }^{(3)}$.

The accuracy of a code calculation can be evaluated through these values, by representing the discrepancies of the addressed calculation with respect to the experimental data with a point in the WFAA plane. The most significant information is given by AA, which represents the relative magnitude of these discrepancies; WF supplies a different information allowing to better identify the character of accuracy. In fact, depending on the transient and on the parameter considered, low frequency errors can be more important than high frequency ones, or vice versa.

(3) : In fact, it really represents the centre of gravity of the amplitude spectrum of Fourier transform of the difference function $\Delta \mathrm{F}(\mathrm{t})$; therefore, it evaluates the kind of error of the addressed calculation (which can be more or less relevant depending on the characteristics of the analyzed transient) 
Trying to give an overall picture of the accuracy of a given calculation, it is required to combine the information obtained for the single parameters into average indexes of performance. This is obtained by defining the following quantities:

$$
\begin{aligned}
& (\mathrm{AA})_{\mathrm{tot}}=\sum_{\mathrm{i}=1}^{\mathrm{N}_{\mathrm{var}}}(\mathrm{AA})_{\mathrm{i}}\left(\mathrm{w}_{\mathrm{f}}\right)_{\mathrm{i}} \\
& (\mathrm{WF})_{\text {tot }}=\sum_{\mathrm{i}=1}^{\mathrm{N}_{\mathrm{var}}}(\mathrm{WF})_{\mathrm{i}}\left(\mathrm{w}_{\mathrm{f}}\right)_{\mathrm{i}}
\end{aligned}
$$

with: $\quad \sum_{i=1}^{N_{\text {var }}}\left(w_{f}\right)_{i}=1$, where:

- $\mathrm{N}_{\mathrm{var}}$ is the number of parameters selected (to which the method has been applied);

- $\left(\mathrm{w}_{\mathrm{f}}\right)_{\mathrm{i}}$ are weighting factors introduced for each parameter, to take into account their importance from the viewpoint of safety analyses.

The need of $\left(\mathrm{w}_{\mathrm{f}}\right)_{\mathrm{i}}$ definition derives from the fact that the addressed parameters are characterized among other things by different importance and reliability of measurement. Thus, each $\left(\mathrm{w}_{\mathrm{f}}\right)_{\mathrm{i}}$ takes into account of:

- "experimental accuracy": experimental measures of thermalhydraulic parameters are characterized by a more or less sensible uncertainty due to:

- intrinsic characteristics of the instrumentation;

- assumptions formulated in getting the measurement;

- un-avoidable discrepancies existing between experimental measures and the code calculated ones (mean values evaluated in cross-sections, volume centers, or across junctions, etc.);

- "safety relevance": particular importance is given to the accuracy quantification of calculations concerned with those parameters (e.g. clad temperature, from which PCT values are derived) which are relevant for safety and design.

Last, a further contribution is included in the weighting factors definition; this is a component aiming at accounting for the physical correlations governing most of the thermalhydraulic quantities. Taking as reference parameter the primary pressure (its measurement can be considered highly reliable), a normalization of the AA values calculated for other parameters with respect to the AA value calculated for the primary side pressure is carried out. Doing thusly, the weighting factor for the generic $j$-th parameter, is defined as:

$$
\left(\mathrm{w}_{\mathrm{f}}\right)_{j}=\frac{\left(\mathrm{w}_{\text {exp }}\right)_{\mathrm{j}} \cdot\left(\mathrm{w}_{\text {saf }}\right)_{\mathrm{j}} \cdot\left(\mathrm{w}_{\text {norm }}\right)_{\mathrm{j}}}{\sum_{\mathrm{j}=1}^{\mathrm{Nar}_{\mathrm{var}}}\left(\mathrm{w}_{\text {exp }}\right)_{\mathrm{j}} \cdot\left(\mathrm{w}_{\text {saf }}\right)_{\mathrm{j}} \cdot\left(\mathrm{w}_{\text {norm }}\right)_{\mathrm{j}}}
$$

and: 


$$
\sum_{j=1}^{N_{\text {var }}}\left(w_{f}\right)_{j}=1
$$

where:

- $\mathrm{N}_{\mathrm{var}}$ is the number of parameters to which the method is applied;

- $\left(\mathrm{w}_{\exp }\right)_{j}$ is the contribution related to the experimental accuracy;

- $\left(\mathrm{w}_{\mathrm{saf}}\right)_{j}$ is the contribution expressing the safety relevance of the addressed parameter;

- $\left(\mathrm{w}_{\text {norm }}\right)_{j}$ is component of normalization with reference to the average amplitude evaluated for the primary side pressure.

This introduces a degree of engineering judgment that has been fixed by a proper and unique definition of the weighting factors ${ }^{14}$ (see Table 1)). The most suitable factor for the definition of an acceptability criterion, therefore, for using the method, is the average amplitude $A A$. With reference to the accuracy of a given calculation, it has been defined the following acceptability criterion:

$$
(A A)_{t o t}<K
$$

where $K$ is an acceptability factor valid for the whole transient. The lower the $(A A)_{\text {tot }}$ value is, the better the accuracy of the analyzed calculation (i.e., the code prediction capability and acceptability is higher). On the other hand, $(A A)_{\text {tot }}$ should not exceed unity in any part of the transient ( $A A=1$ means a calculation affected by a $100 \%$ error). Because of this requirement, the accuracy evaluation should be performed at different steps during the transient.

With reference to the experience gathered from previous application of this methodology, $K=0.4$ has been chosen as the reference threshold value identifying good accuracy of a code calculation. In addition, in the case of upper plenum pressure, the acceptable threshold is given by $K=0.1$.

Table 1. Selected weighting factor components for typical thermalhydraulic parameters.

\begin{tabular}{|l|c|c|c|c|}
\hline \multicolumn{1}{|c|}{ Parameter } & ID & $\mathbf{w}_{\mathbf{e x p}}$ & $\mathbf{w}_{\text {Saf }}$ & $\mathbf{w}_{\text {norm }}$ \\
\hline Primary pressure & PP & 1.0 & 1.0 & 1.0 \\
\hline Secondary pressure & SP & 1.0 & 0.6 & 1.1 \\
\hline Pressure drops & PD & 0.7 & 0.7 & 0.5 \\
\hline Mass inventories & MS & 0.8 & 0.9 & 0.9 \\
\hline Flow rates & FR & 0.5 & 0.8 & 0.5 \\
\hline Fluid temperatures & FT & 0.8 & 0.8 & 2.4 \\
\hline Clad temperatures & CT & 0.9 & 1.0 & 1.2 \\
\hline Collapsed levels & LV & 0.8 & 0.9 & 0.6 \\
\hline Core power & PW & 0.8 & 0.8 & 0.5 \\
\hline
\end{tabular}




\section{Characterization of the Ranges of Variation of the Input Uncertain Parameters by FFTBM}

\subsection{The FFTBM procedure}

A procedure to characterize the boundaries of the input uncertain parameters shall give emphasis to the connection between the 'objective sources' of uncertainty ${ }^{9}$ and the list of input uncertain parameters.

It is worth noting that 'objective sources' of uncertainty and 'suitable lists' of input uncertain parameters should be considered for uncertainty method design and application, respectively. Moreover, both sets of parameters (i.e. 'objective sources' of uncertainty and 'suitable lists') are part of recognized international documents. Namely, reference is made for the sources of uncertainty to an OECD/CSNI (Organization for Economic Cooperation and Development / Committee on the Safety of Nuclear Installations) document issued in 1998 and to a more recent IAEA (International Atomic Energy Agency) document, issued in 2008. The lists of input uncertain parameters are derived from the application of various uncertainty methods in the UMS ${ }^{5}$ and BEMUSE projects ${ }^{15}$ completed or nearly completed under the umbrella of OECD/CSNI.

The proposed procedure is based on the use of the FFTBM plus series of considerations as the one here below:

a) One single input parameter shall not be 'responsible' for the entire error |exp-calc|, unless exceptional situations to be evaluated case by case;

b) Initial guess for Max and Min for variation ranges have to be based on the usual (adopted) expertise;

c) More than one experiment can be used per each NPP and each scenario. Highly influential parameters are expected to be the same. The bounding ranges should be considered for the NPP uncertainty analysis;

d) A data base of suitable uncertainty input parameters can be created per each NPP and each transient scenario.

Once the facility and the experiment has been chosen based on the selected NPP transient, the main steps of the procedure proposed in Figure 5 are:

- To run the Reference Case (RC),

- To select the Responses (R),

- To derive the $\mathrm{AA}_{\mathrm{R}}^{\mathrm{REF}}$ for each selected response by FFTBM,

- To select a set of Input Uncertainty Parameters (IP),

- To run sensitivity cases for which identified input parameter,

- To apply FFTBM to the sensitivity cases to obtain $\mathrm{AA}_{\mathrm{R}}^{*, \mathrm{IP}}$,

- To apply the criteria for identifying the range [Min, Max],

- To discard not relevant Input Uncertainty Parameters.

About the criteria to be applied for the identification of the range of the input parameters, seven different criteria are under investigation to test the procedure. Each criterion includes a set of statements to be satisfied. The seven proposed criteria are listed hereafter: 


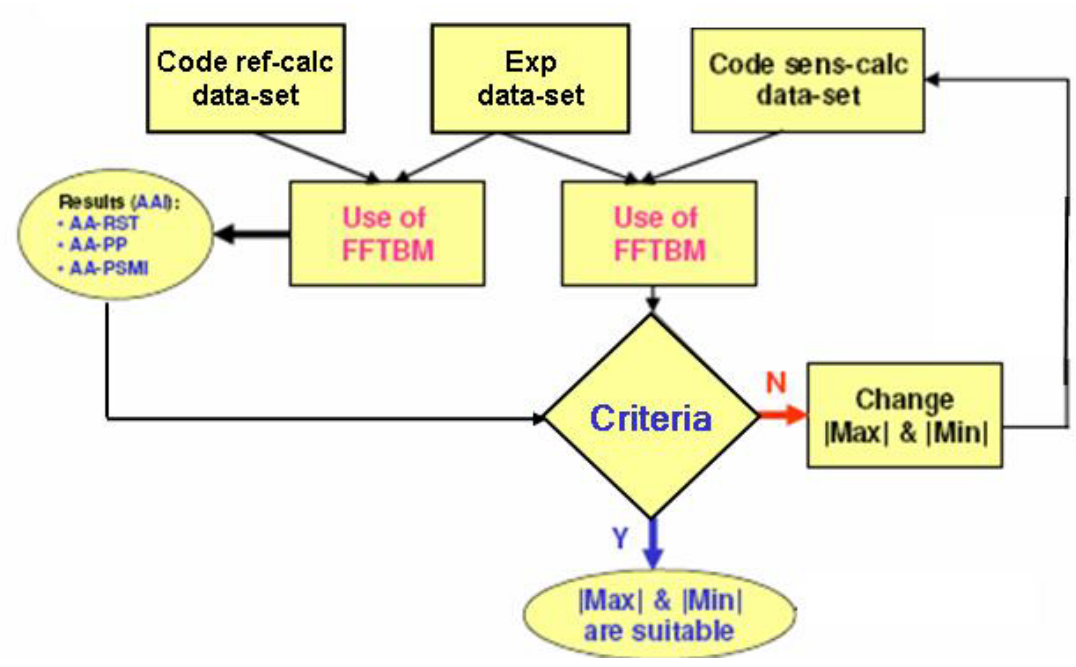

Figure 5. FFTBM procedure for identifying the range of variation of input uncertain parameters.

CR1: CR1.a: $\mathrm{AA}_{\mathrm{p}}^{*}, \mathrm{IP}<0.1$ p: pressure

CR1.b: $\operatorname{MAX}\left(\mathrm{AA}_{\mathrm{R}_{\mathrm{i}}}^{*, \mathrm{IP}} / \mathrm{AA}_{\mathrm{R}_{\mathrm{i}}}^{\mathrm{REF}}\right)-1<0 \quad \mathrm{i}=1, \ldots, \mathrm{N} \quad \mathrm{N}$ responses

CR2: CR2.a: $\mathrm{AA}_{\mathrm{p}}^{*}$,IP $<0.1$

CR2.b: $\mathrm{AA}_{\mathrm{G}}^{*, \mathrm{IP}} / \mathrm{AA}_{\mathrm{G}}^{\mathrm{REF}}-1<\mathrm{T} 1 \quad \mathrm{AA}_{\mathrm{G}}^{*}, \mathrm{IP}=\sqrt{\sum_{\mathrm{i}=1}^{\mathrm{N}}\left(\mathrm{AA}_{\mathrm{R}_{\mathrm{i}}}^{*, \mathrm{IP}}\right)^{2}} \quad$ T1: Acceptability threshold \#1

CR2.c: $\operatorname{MAX}\left(\mathrm{AA}_{\mathrm{R}_{\mathrm{i}}}^{*, \mathrm{IP}} / \mathrm{AA}_{\mathrm{R}_{\mathrm{i}}}^{\mathrm{REF}}\right)-1<\mathrm{T} 2 \quad \mathrm{~T} 2$ : Acceptability threshold \#2

CR3: CR3.a: $\mathrm{AA}_{\mathrm{p}}^{*}, \mathrm{IP}<0.1$

CR3.b: $\mathrm{AA}_{\mathrm{G}}^{*, \mathrm{IP}} / \mathrm{AA}_{\mathrm{G}}^{\mathrm{REF}}-1<\mathrm{T} 1 \quad \mathrm{AA}_{\mathrm{G}}^{*, \mathrm{IP}}=\sqrt[N]{\prod_{\mathrm{i}=1}^{\mathrm{N}} \mathrm{AA}_{\mathrm{R}_{\mathrm{i}}}^{*, \mathrm{IP}}}$

CR3.c: $\operatorname{MAX}\left(\mathrm{AA}_{\mathrm{R}_{\mathrm{i}}}^{*}, \mathrm{IP} / \mathrm{AA}_{\mathrm{R}_{\mathrm{i}}}^{\mathrm{REF}}\right)-1<\mathrm{T} 2$ 
CR4: CR4.a: $\mathrm{AA}_{\mathrm{p}}^{*}$,IP $<0.1$

CR4.b: $\mathrm{AA}_{\mathrm{G}}^{*, \mathrm{IP}} / \sqrt{\mathrm{N}}-1<\mathrm{T} 1 \quad \mathrm{AA}_{\mathrm{G}}^{*}, \mathrm{IP}=\sqrt{\sum_{\mathrm{i}=1}^{\mathrm{N}}\left(\mathrm{AA}_{\mathrm{R}_{\mathrm{i}}}^{*, \mathrm{IP}} / \mathrm{AA}_{\mathrm{R}_{\mathrm{i}}}^{\mathrm{REF}}\right)^{2}}$

CR5: CR5.a: $\mathrm{AA}_{\mathrm{p}}^{*}, \mathrm{IP}<0.1$

CR5.b: $\mathrm{AA}_{\mathrm{G}}^{*}, \mathrm{IP}-1<\mathrm{T} 1$

$$
\mathrm{AA}_{\mathrm{G}}^{*, \mathrm{IP}}=\sqrt{\frac{\sum_{\mathrm{i}=1}^{\mathrm{N}}\left(\mathrm{AA}_{\mathrm{R}_{\mathrm{i}}}^{*, \mathrm{IP}}\right)^{2}}{\sum_{\mathrm{i}=1}^{\mathrm{N}}\left(\mathrm{AA}_{\mathrm{R}_{\mathrm{i}}}^{\mathrm{REF}}\right)^{2}}}
$$

CR6: CR6.a: $\mathrm{AA}_{\mathrm{p}}^{*, \mathrm{IP}}<0.1$

CR6.b: $\mathrm{AA}_{\mathrm{G}}^{* \mathrm{IP}} / \mathrm{AA}_{\mathrm{G}}^{\mathrm{REF}}-1<\mathrm{T} 1 \quad A A_{G}^{*, I P}=\frac{1}{N} \cdot \sum_{i=1}^{N} A A_{R}^{*, I P}$

CR7: CR7.a: $\mathrm{AA}_{\mathrm{p}}^{*}$,IP $<0.1$

CR7.b: $\mathrm{AA}_{\mathrm{G}}^{*}, \mathrm{IP}-1<\mathrm{T} 1$

$$
A A_{G}^{*, I P}=\sqrt{\frac{\sum_{i=1}^{N}\left(A A_{R_{i}}^{*, I P} \cdot w_{f_{i}}\right)^{2}}{\sum_{i=1}^{N}\left(A A_{R_{i}}^{R E F} \cdot w_{f_{i}}\right)^{2}}}
$$

$w_{f_{i}}$ FFTBM weighting factors (see Eq. 15 and Table 1)

\subsection{Sample applications of FFTBM procedure}

The FFTBM procedure has been applied to three tests so far:

1. Marviken Test CFT04,

2. Edwards pipe,

3. LOBI tests A1-83 (analysis currently on going).

In this section the summary of the results achieved for the "Edwards pipe" application is provided. The sketch of the nodalization and of the boundary conditions of the experiment are given in Figure 6. The responses of interest are the pressure and the void fraction measured at about middle length of the pipe. The input parameters that have been initially investigated were about 10. Through the use of the FFTBM, the input parameters that determine a little variation of AA values in correspondence of large variations of those parameters were discharged (e.g. the pipe roughness and the thermal non-equilibrium constant in the Henry-Fauske critical flow model). Finally the analysis was reduced to investigate the following input parameters:

1. The form loss coefficient (Kloss),

2. The initial fluid temperature, 


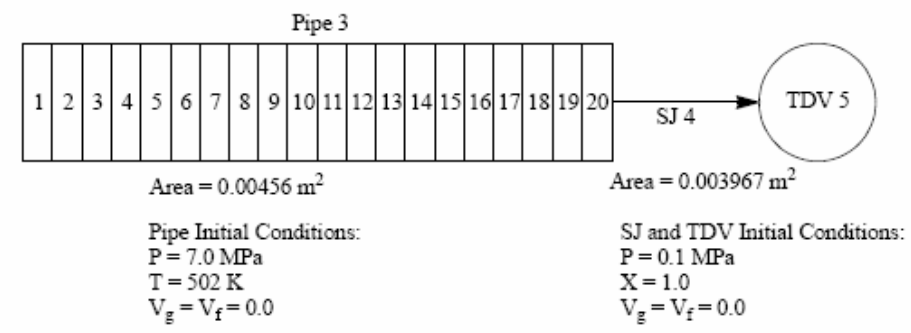

Figure 6. Nodalization and Boundary condition of the Edward pipe.

3. The break area,

4. The "Henry-Fauske" choked flow model, discharge coefficient.

Figure 7 shows the results of the application of the FFTBM procedure in relation with the statement ' $a$ ' of each criteria (i.e. $\mathrm{AA}_{\mathrm{p}}^{*}$,IP $<0.1$ ) for the four selected input parameters. The attention shall be focused on the AA value for the pressure $\left(\mathrm{AA}_{\mathrm{P}}\right)$ and its limit equal to 0.1. The corresponding ranges of variation [Min(IP), Max(IP) $]_{a}$ for each of the four input parameters satisfying the statement 'a' are then identified.
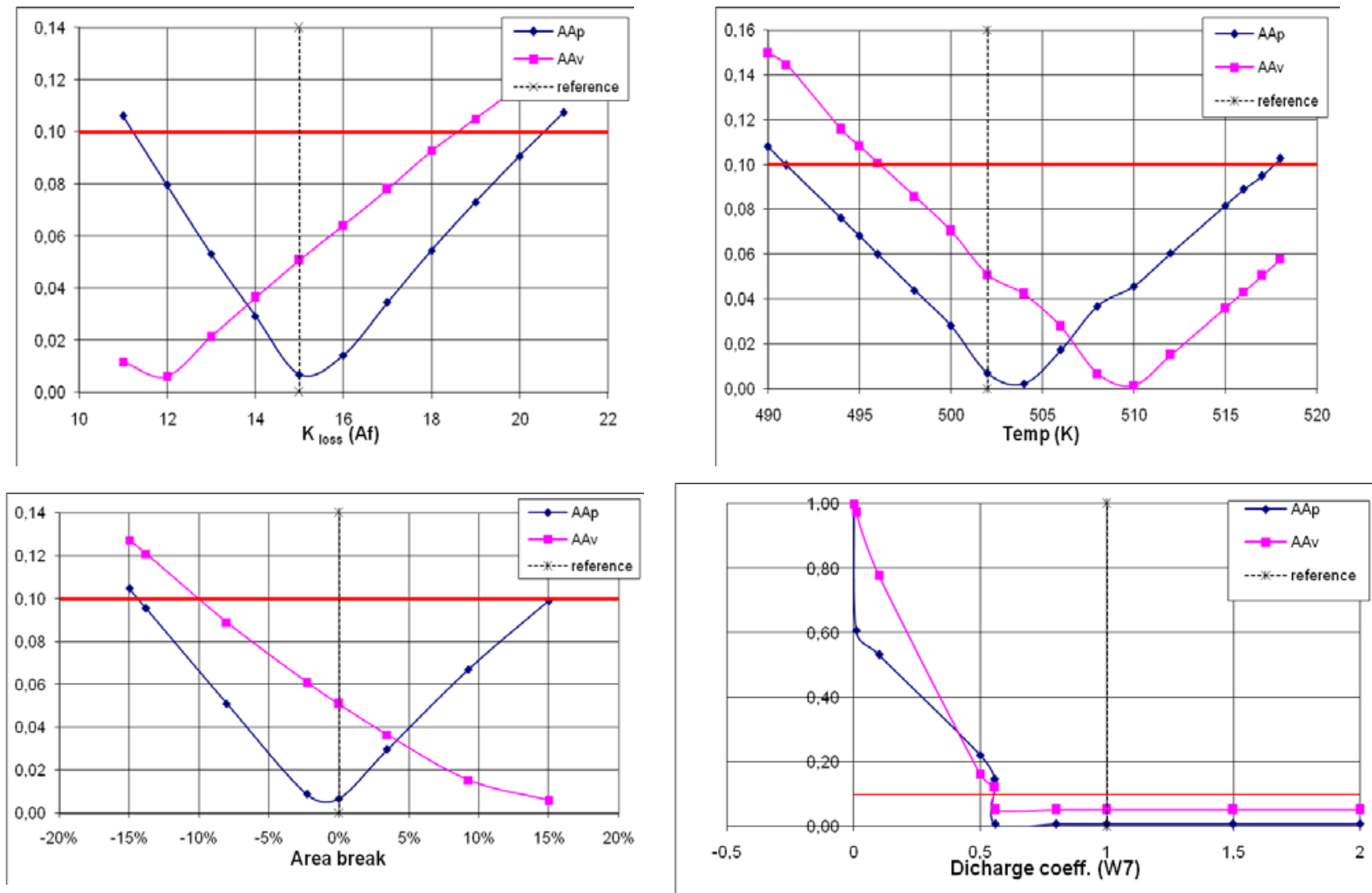

Figure 7. Values of $\mathrm{AA}_{\mathrm{P}}$ and ranges of variation of input parameters. 
In order to consider the overall accuracy of a calculation, one of the seven criteria proposed in section 5.1 can be applied. The results are shown in Figure 8 together with different values for the acceptability thresholds T. It shall be noted that the y-axis represents the values of the left side of the inequalities and that the statement criteria CR1.b is the same of the statements CR2.c and CR3.c. The corresponding ranges of variation $[\mathrm{Min}(\mathrm{IP}), \operatorname{Max}(\mathrm{IP})]_{\mathrm{b}, \mathrm{c}}$ for each of the four input parameters satisfying the statements ' $b$ ' and ' $c$ ' are then identified. The final range of variation [Min(IP), Max(IP)] for each input parameter is then calculated by the intersection of the ranges of variation derived for the statements ' $a$ ', ' $b$ ' and ' $c$ ' for each criterion.
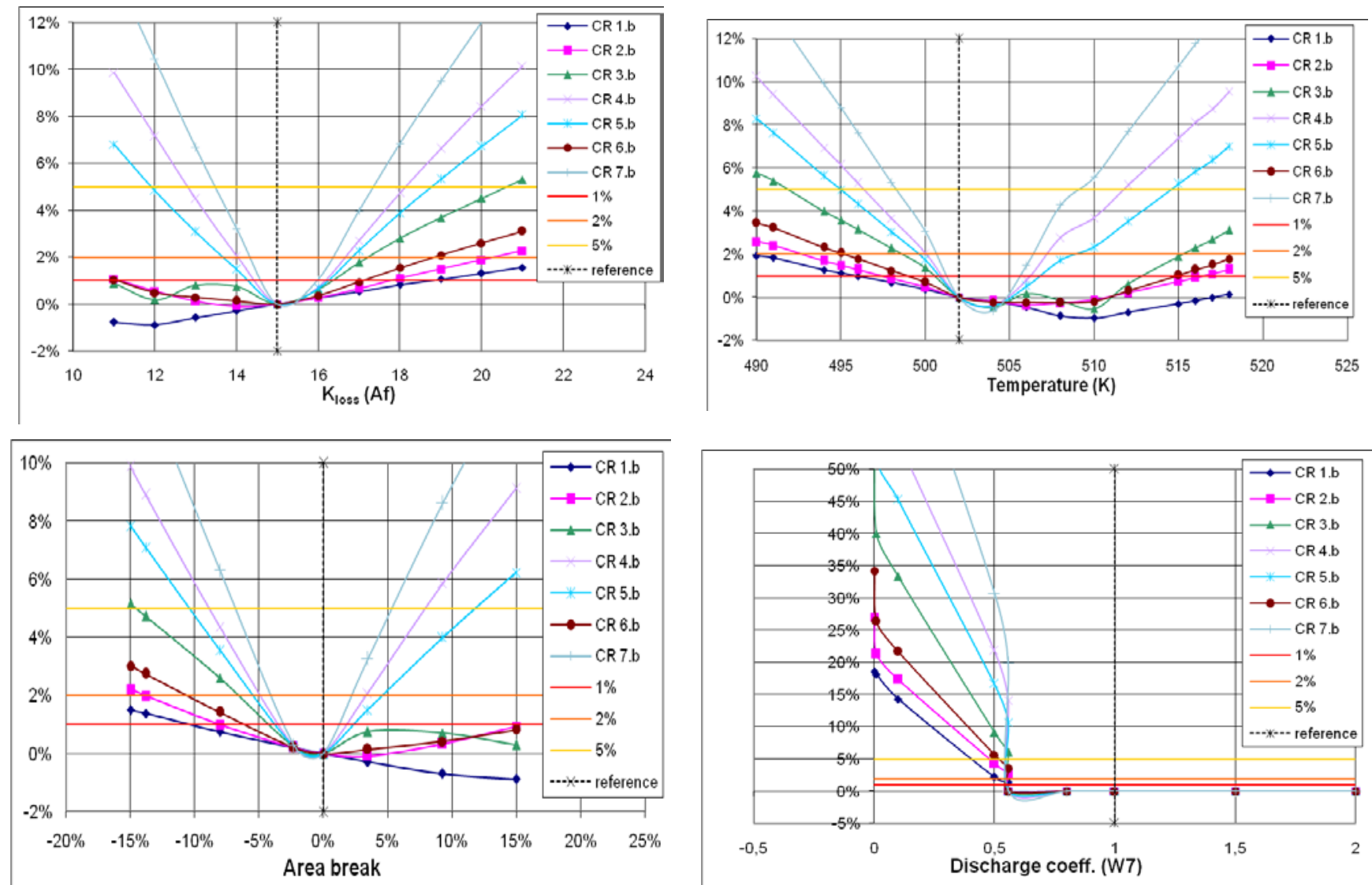

Figure 8. Results of the FFTBM procedure and ranges of variation of input parameters depending on different criteria.

\section{Conclusions}

The FFTBM allows a quantitative judgment for a given calculation. Each set of two curves constituted by a calculated and a measured time trend can be processed by FFTBM. The transformation from time to the frequency domain avoids the dependence of the error from the transient duration. Weight factors are attributed to each time trend to make possible the summing up of the error and the achievement of a 
unique threshold for accepting a calculation. The quantitative accuracy evaluation by FFTBM must be carried out following the demonstration that the calculation is qualitatively acceptable.

The objective of the paper is to propose a FFTBM-based procedure to characterize the ranges of variation of the input uncertain parameters in place of the usual approach based (mostly) on engineering judgment for the uncertainty methods based on the propagation of input error. The application to a simplified problem like Edwards pipe has been discussed.

\section{References}

[1] Leonardi M., D'Auria F., Pochard R., "The FFT based method in the frame of the UMAE", Spec. Workshop on Uncertainty Analysis Methods, London, March 1994.

[2] Ambrosini W., Bovalini R., D'Auria F., "Evaluation of accuracy of thermalhydraulic code calculations", J. Energia Nucleare,vol. 2, 1990.

[3] Riebold W., "Minutes of the OECD/CSNI SACTE Task Group Meeting", Paris, December 1987.

[4] Pochard R., Porracchia A., "Assessment closure proposal", OECD/CSNI SACTE Task Group Meeting, Paris, December 1986.

[5] Wickett T., D’Auria F., Glaeser H., Chojnacki E., Lage C., Sweet D., Neil A., Galassi G. M., Belsito S., Ingegneri M., Gatta P., Skorek T., Hoper E., Kloos M., Ounsy M. and Sanchez J. I., "Report of the Uncertainty Method Study for Advanced Best Estimate Thermalhydraulic Code Applications”, OECD/NEA/CSNI R (97) 35, I and II, June 1998.

[6] Wilks S.S. Determination of sample sizes for setting tolerance limits, J. Ann. Math. Statist. 12, pp 91-96, 1941.

[7] D’auria F., Debrecin N., Galassi G. M., "Outline of the Uncertainty Methodology based on Accuracy Extrapolation (UMAE)", J. Nuclear Technology, 109, No 1, pp 21-38 (1995).

[8] D'auria F., Giannotti W., "Development of Code with capability of Internal Assessment of Uncertainty”, J. Nuclear Technology, 131, No. 1, pp 159-196 (2000).

[9] Petruzzi A., D’Auria F., "Thermal-Hydraulic System Codes in Nuclear Reactor safety and Qualification procedures”, Science and Technology of Nuclear Installations, ID 460795, 2008.

[10] Ambrosini W., Bovalini R. and D'Auria F., Evaluation of Accuracy of Thermal-hydraulic Codes Calculations, J. En. Nucl. 7, 1990.

[11] D’Auria F. and Galassi G.M., Code Validation and Uncertainties in System Thermal-hydraulics, J. Prog. in Nucl. En., 33, pp 175-216, 1998.

[12] Bovalini R. and D'Auria F., Scaling of the accuracy of Relap5/mod2 Code J. Nucl. Eng. \& Des. 139, pp 187-204, 1993.

[13] Brigham E. O., "The Fast Fourier Transform", Prentice Hall, Englewood Cliffs (NJ), 1974.

[14] D'Auria F., Leonardi M., Pochard R., "Methodology for the evaluation of thermahydraulic codes accuracy", Int. Conf. on 'New Trends in Nuclear System Thermohydraulics', Pisa, May 30 - June 2, 1994.

[15] OECD/NEA/CSNI, "BEMUSE Programme. Phase 3 report <Uncertainty and Sensitivity Analysis of the LOFT L2-5 experiment>”, OECD/CSNI Report NEA/CSNI/R(2007)4. 